\title{
Estrategias para el abordaje de las Teorías del Desarrollo Humano
}

\author{
Strategies for addressing the Theories of Human Development
}

\author{
Dinamarca González \\ dinamarkgr@gmail.com \\ Código ORCID: 0000-0002-4400-1377 \\ Universidad Pedagógica Experimental Libertador, Venezuela
}

Recibido: Abril 2019 / Arbitrado: Mayo 2019 / Publicado: Julio 2019

RESUMEN

La presente investigación tuvo como propósito identificar las estrategias utilizadas por los docentes en los cursos que abordan los contenidos relacionados con las teorías y postulados del desarrollo humano. El enfoque utilizado fue el cuantitativo con un diseño de campo. La muestra estuvo conformada por 15 estudiantes de la especialidad Educación Inicial de la Universidad Pedagógica Experimental Libertador, Miranda, Venezuela. La técnica fue la observación y el instrumento el cuestionario. Los resultados evidenciaron que existe desconocimiento sobre el diseño de estrategias de enseñanza por parte de quienes imparten los contenidos, postulados y elementos de las teorías del desarrollo humano. En tal sentido, se requiere del diseño de estrategias basadas en la enseñanza estratégica para favorecer el ejercicio de la docencia y el aprendizaje de los estudiantes, quienes serán docentes de los primeros niveles del sistema educativo

Palabras clave:

Estrategias de enseñanza; teorías del desarrollo humano; Educación Inicial

ABSTRACT

The purpose of this research was to identify the strategies used by teachers in courses that address content related to theories and postulates of human development. The approach used was quantitative with a field design. The sample consisted of 15 students from the Initial Education specialty of the Libertador Experimental Pedagogical University, Miranda, Venezuela. The technique was observation and the instrument was the questionnaire. The results evidenced that there is ignorance about the design of teaching strategies by those who teach the content, postulates and elements of the theories of human development. In this sense, the design of strategies based on strategic teaching is required to favor the exercise of teaching and learning of students, who will be teachers of the first levels of the educational system. 


\section{INTRODUCCIÓN}

La importancia que las teorías psicológicas relativas al desarrollo humano tienen para el docente de cualquier especialidad es incuestionable. Muestra de ello es que los diseños curriculares y programas de los diferentes cursos de formación académica en las instituciones universitarias encargadas de formar docentes las contemplan como esencial.

La profesión docente resulta ser una de las más complejas debido al hecho de que los resultados de su ejercicio impactan de manera permanente, negativa o positivamente, en las vidas de seres humanos que se forman. Es por esto que el docente en formación debe procurar una instrucción académica y profesional completa y de calidad, que responda a las actuales necesidades de la población que atenderá según su especialidad, pues este tendrá a su cuidado y bajo su responsabilidad la formación a las siguientes generaciones de ciudadanos; por tal razón, es imprescindible que su educación de calidad le permita ejercer satisfactoriamente la docencia de manera que derive en el desarrollo armónico e ideal de cada uno de sus educandos.

En este camino hacia su formación académica y profesional, el estudiante de la carrera docente, sobre todo el de la especialidad de Educación Inicial, se encontrará con cursos en los cuales, el estudio acerca de las teorías del desarrollo humano, son esenciales. No obstante, durante mucho tiempo estas teorías han sido subestimadas o inadecuadamente trabajadas.

La importancia que las teorías psicológicas del desarrollo humano tienen para el docente de cualquier especialidad es evidente; más aún lo son para los docentes de los primeros niveles del sistema educativo. La situación de desconocimiento de los contenidos, postulados y elementos fundamentales de las teorías del desarrollo humano viene constituyendo un serio problema por ser obstáculo en la formación de un profesional de la docencia que pretenda además desempeñarse en la fase de Educación Inicial.
En atención a lo anterior, la especialidad Educación Inicial del Instituto Pedagógico de Miranda José Manuel Siso Martínez (IPMJMSM) de la Universidad Pedagógica Experimental Libertador (UPEL), incluye en su matriz curricular, cursos correspondientes a los Componentes de Formación Especializada y de Formación Pedagógica, los cuales tienen como eje fundamental el análisis y el estudio de las teorías del desarrollo humano, por ser la base de los procesos de enseñanza y de aprendizaje para quienes están formándose como educadores de niños en los primeros años de vida.

Si bien es cierto que para la carrera docente el manejo de estas teorías es necesario; para la especialidad Educación Inicial resulta imprescindible y vital, razón por la cual, en la gran mayoría de los cursos de la especialidad, tales teorías constituyen un eje transversal que permea todos los contenidos de las mismas.

Es así como en el presente estudio, al hacer referencia a la psicología del desarrollo humano y el abordaje de las teorías, no se está haciendo mención a la psicología como un curso específico, sino más bien a los elementos teóricos que la constituyen como un eje fundamental común en todos los cursos del componente de Formación Especializada en la especialidad Educación Inicial en el IPMJMSM e incluso del componente de Formación Pedagógica de la carrera en el Instituto.

Lo anterior se fundamentó de manera más detallada en el marco referencial de este estudio. Un ejemplo radica en las ideas de Pérez Lo Presti (2007), quien afirma que el docente debe conocer los alcances de la psicología y sus enunciados; pero debe comprender que por encima de todo, existe una aplicabilidad y grado de praxis que le ha de permitir no solo llevarlo al plano laboral en su trabajo con los estudiantes, sino que adicionalmente le permitirá aplicarlo en su crecimiento personal, al darle herramientas para conocerse y conocer al otro, traduciéndose en logros integrales. Por tales razones, la 
especialidad Educación Inicial en el IPMJMSM no solo no escapa de esta necesidad de conocer y profundizar en los elementos teóricos mencionados, sino que sus cimientos se hallan en la psicología y su praxis determinada por esta, por cuanto constituye el eje y la brújula de este nivel educativo.

Para Pérez Lo Presti (2007) penetrar en los estudios de los postulados teóricos que devienen de la psicología ha de permitir al docente en formación y en ejercicio, "adquirir un bagaje de conocimientos que le ayudará a detectar y canalizar aquellos alumnos que presenten actitudes disfuncionales en la dinámica del salón de clases" (p. 627). Además permite al docente la intervención oportuna y la mediación para favorecer el desarrollo integral del niño, llevar a cabo una evaluación y planificación de la enseñanza y de las diferentes situaciones de aprendizaje, en concordancia con las pautas evolutivas del desarrollo infantil y de acuerdo con los parámetros que los enfoques teóricos han determinado como producto de largos años de estudios y de comprobaciones científicas.

En consecuencia, la presente investigación tuvo como propósito identificar las estrategias utilizadas por los docentes de la especialidad Educación Inicial del Instituto Pedagógico de Miranda José Manuel Siso Martínez en los cursos que abordan los contenidos relacionados con las teorías y postulados del desarrollo humano (González, 2014).

\section{El proceso de enseñanza}

El proceso de enseñanza, fundamentado teóricamente en el cognitivismo, según refiere Hernández (1998) apoyado en las ideas de Ausubel (1976), debe estar orientado al logro de aprendizajes significativos y al desarrollo de habilidades estratégicas generales y específicas de aprendizaje (p. 133). Tal afirmación será la premisa que oriente el presente estudio de principio a fin.

\section{Las estrategias}

De acuerdo con Flores (2002), pueden considerarse sinónimo de procedimiento y también definirse como un conjunto de acciones ordenadas dirigidas a la consecución de una meta. Los procedimientos 0 estrategias constituyen el saber hacer, el saber actuar en forma eficaz (p. 27). Visto desde esta perspectiva, todo lo que se hace en la vida cotidiana con un propósito específico implica el uso de estrategias, aún de manera inconsciente.

La utilización del término aplicado de manera específica al ámbito educativo es reciente, de acuerdo con Esteban y Zapata (2008). Este se incorpora al ámbito de la psicología del aprendizaje y a la educación, como una forma más de resaltar el carácter procedimental que tiene todo aprendizaje y que encaja perfectamente con la psicología cognitiva, con la perspectiva constructivista del conocimiento y el aprendizaje (p. 6), esto con el respectivo valor que se le asigna a los elementos tácticos en el proceso de construcción de conocimientos.

\section{Estrategias de enseñanza}

Según Cueva (2000) una estrategia de enseñanza es la secuencia de actividades conscientes, estructuradas bajo procedimientos y recursos en un plan deliberado controlado por el docente para promover aprendizajes significativos; orientan la enseñanza del profesor. En ella es necesario precisar los métodos de enseñanza, pues ocupan un lugar medular en su preparación y ejecución. Si se analiza en detalle esta definición de estrategia de enseñanza y se compara con la definición que Acevedo (2002) hace del término enseñanza, pueden establecerse puntos de encuentro: el rol del docente o facilitador, la intencionalidad de las acciones, la consideración de los procesos de enseñanza y de aprendizaje y la atención a elementos metodológicos fundamentales. 
Lo anterior permitió a la autora del presente estudio asumir dichas definiciones en posteriores consideraciones, en función de interpretar que existe una estrecha relación entre ambos términos fundamentada en la cohesión que Acevedo (ob. cit.) afirma que existe entre dichos procesos.

Según Pozo y Monereo (1999), la estrategia está formada por técnicas y métodos en un orden jerárquico donde la estrategia es de mayor complejidad o amplitud, seguida del método y finalmente la técnica.

\section{Métodos de enseñanza}

La palabra método es definida por la Universidad Nacional Abierta (1983), como el camino para llegar a un lugar determinado, por lo cual en lo referente a método de enseñanza, este viene a ser el camino, el modo o la forma de enseñar o guiar la práctica educativa, la situación deenseñanza.

De acuerdo con lo que el antiguo Ministerio de Educación (1987) presenta en el Manual del Docente, los métodos son procedimientos generales basados en principios lógicos que pueden ser comunes a varias ciencias.

Es así como se puede afirmar que de acuerdo con algunos planteamientos de la didáctica, los métodos obedecen a principios que permiten que estos puedan ser clasificados de acuerdo a diferentes criterios, en función de que puedan favorecer una visión global de los distintos métodos de enseñanza y técnicas.

Conviene destacar que al presentar una clasificación de los métodos de enseñanza, esta clasificación puede obedecer a los intereses y visión del investigador. Por tanto, para efectos del presente estudio se ha recurrido a una forma de clasificación sencilla y bastante conocida.

\section{Las técnicas de enseñanza}

La Universidad Nacional Abierta (1983) define la palabra técnica como "el cómo hacer algo" por lo que la técnica de enseñanza es definida como el procedimiento que se adopta para orientar las actividades del docente y del estudiante durante los procesos de enseñanza y de aprendizaje.

En tal sentido y, teniendo en consideración la afirmación anterior, la autora considera que las técnicas se convierten en formas específicas usadas de manera idónea, determinada y particular para promover el logro de una objetivo o competencia en una situación de enseñanza.

Igual que los métodos de enseñanza, las técnicas también cuentan con diversas clasificaciones según diversos criterios. Para efectos de la presente investigación se tomará en consideración la clasificación que la Universidad Nacional Abierta (1983) propone (ver Tabla 1).

Tabla 1. Técnicas de enseñanza

\begin{tabular}{ll}
\hline Criterios de clasificación (centrados en) & Técnicas de enseñanza \\
\hline 1.- El docente & $\begin{array}{l}\text { a) exposición } \\
\text { b) Demostración } \\
\text { c) Pregunta }\end{array}$ \\
2.- El estudiante & a) Socializadas: \\
& a.1) Debate dirigido \\
& a.2) Discusión en pequeños grupos \\
& a.3) Phillip 66 \\
a.4) Phillip 22 o cuchicheo & a.5) Torbellino o lluvia de ideas
\end{tabular}



a.6) Dramatización
a.7) Juegos didácticos
a.8) Foro
a.9) Panel
a.10) Seminario
b) Individualizadas
b.1) Guías
b.2) Fichas
b.3) Estudio dirigido

Fuente: Universidad Nacional Abierta (1983). Técnicas de enseñanza.

\section{La formación del docente de Educación Inicial}

La formación académica del docente encargado de trabajar con los niños más pequeños del Sistema Educativo Venezolano debe contemplar toda una gama de contenidos y prácticas que permitan, al educador en formación, desarrollar y potenciar las competencias que garanticen la construcción del perfil que corresponde al docente idóneo para la educación.

En tal sentido, la Universidad Pedagógica Experimental Libertador (UPEL) como primera institución garante de la formación de docentes a nivel nacional prevé en su diseño curricular elementos claves para garantizar la formación del docente de la especialidad Educación Inicial.

En el apartado correspondiente a la Fundamentación del Diseño Curricular de la Especialidad de Educación Preescolar de la Universidad Experimental Libertador -UPEL (1996a), se afirma:

Si consideramos que los primeros años de vida del individuo son determinantes para su desarrollo integral, es fundamental asignarle un papel relevante a la formación del docente para el primer nivel del sistema educativo: ...Estudiosos de la infancia e investigadores de la educación preescolar sostienen que el docente para este nivel debe poseer un dominio profundo de la Psicología infantil y una preparación sólida en los principios que orientan la acción educativa para el logro de los objetivos correspondientes a las áreas de desarrollo físico, cognoscitivo, socio-afectivo y psicomotor

...Asimismo debe utilizar los medios y recursos más adecuados a la capacidad de abstracción del niño y a las habilidades específicas que lo caracterizan tomando en cuenta sus intereses y necesidades. (p. 7)

Dentro de las finalidades de la formación del docente de la especialidad Educación Preescolar de la UPEL, en su Diseño Curricular se contempla un plan de estudio integrado adecuado a los lineamientos de las políticas de formación docente y a las orientaciones de la Pedagogía Infantil que garantiza al docente una formación integral de acuerdo con las necesidades del desarrollo nacional y el progreso científico y tecnológico, en aras de hacer posible "una educación integral...centrada en los procesos que exige el desarrollo integral del niño" (p. 8). Al menos éste es el deber ser contemplado en el citado documento, y es responsabilidad de las autoridades, del personal docente y del 
estudiantado velar porque se dé un pleno cumplimiento.

Del mismo modo están plasmados en el documento los objetivos que persigue la especialidad Educación Inicial. Para los fines de este estudio, se han de considerar como claves los siguientes:

- Formar un profesional idóneo: de la docencia, la administración, promoción social e investigación en el nivel de Educación Inicial.

- Formar un profesional de la docencia que brinde al niño en edad preescolar una atención integral.

- Que el egresado de la especialidad Educación Inicial sea un docente competente para aportar alternativas de solución a problemas escolares, familiares, comunales que redunden en beneficio del niño, la familia y la comunidad.

- Que el egresado de la especialidad Educación Inicial sea un docente apto para diseñar y aplicar estrategias y utilizar recursos para el aprendizaje en función de las metas propuestas, de acuerdo a las características biopsicosociales del niño.

Estos objetivos establecidos en el diseño curricular de la especialidad Educación Inicial, constituyen el pilar de la formación de un docente especialista en el área y el logro de los mismos contribuyen a garantizar la idoneidad y calidad de la formación del profesional egresado de la UPEL en dicha especialidad.

La relación de estos objetivos con el propósito de este estudio se corresponden plenamente, en virtud de que el conocimiento y manejo adecuado de las teorías del desarrollo humano y de sus postulados constituyen una parte fundamental por cuanto, además de permitirle al docente conocer al niño y la niña en sus primeros años de vida en cada una de las áreas de su desarrollo evolutivo, le ofrece al docente las herramientas necesarias para poder brindar a sus estudiantes una atención integral de calidad, favoreciendo la adecuada selección de métodos, técnicas, actividades y recursos plenamente ajustados a las necesidades, intereses y potencialidades de los niños en correspondencia con las pautas evolutivas que los estudiosos del desarrollo humano han dejado como legado. Esto también se corresponde con la finalidad y objetivos que están establecidos en el Diseño Curricular del Componente de Formación Especializada de la especialidad Educación Preescolar (UPEL, 1996b). Igualmente, este diseño especifica como finalidad de la especialidad, la formación de un docente que atienda integralmente el proceso de desarrollo del niño, brindando apoyo pedagógico para favorecer los aprendizajes y garantizándole atención a la salud, nutrición desarrollo psicológico y recreación del niño (s.n.).

El curso denominado Desarrollo Cognoscitivo del Niño de 0-7 años en el IPMJMSM código DCN0613, es un curso homologado obligatorio del área de Psicología y Desarrollo Integral, administrado por el Departamento de Psicología del IPMJMSM para la especialidad Educación Inicial de la Universidad Pedagógica Experimental Libertador. Pertenece a un nivel de profundización en la carrera y especialidad, es administrado bajo la modalidad presencialquincenal $y$ en virtud de ello consta de sesiones presenciales y asesorías intersemanales, posee como pre-requisito al curso Psicología Evolutiva (PEV0213).

El curso Desarrollo Cognoscitivo contempla como objetivo general "Analizar los procesos que se encuentran presentes en la adquisición del desarrollo cognoscitivo del niño de 0-7 años, a la luz de las teorías que explican dicho proceso, con el propósito de diseñar, aplicar y evaluar las estrategias necesarias que requiera la intervención educativa" (UPEL, 1996b, p. 2).

Para alcanzar el objetivo general planteado en el anterior párrafo se proponen 
en el programa sinóptico del curso los siguientes objetivos específicos:

- Identificar las diversas teorías que explican el proceso del desarrollo cognoscitivo en el niño de 0-7 años.

- Reconocer las características de los diferentes estudios de los períodos sensorio motriz y preoperacional de la teoría piagetana.

- Valorar los tipos de conocimientos relacionados con los procesos psicológicos que conducen a las nociones y operaciones cognitivas, acompañadas del respectivo diseño de estrategias de intervención pedagógica.

- Diseñar un plan implementando intervenciones pedagógicas que favorezcan en el niño, los procesos del desarrollo cognoscitivo con sus respectivos instrumentos evaluativos.

De acuerdo con el programa aprobado por la Unidad de Currículo de la institución, el curso pretende brindarle al educador en formación, las herramientas necesarias para que este desarrolle las habilidades y destrezas que requiere el abordaje del desarrollo cognoscitivo del niño de educación inicial, de acuerdo con los postulados de Ausbel, Piaget, Vigostky y otros teóricos de renombre en el ámbito educativo y de la psicología del desarrollo humano.

\section{MÉTODO}

En este estudio se utilizó la metodología cuantitativa para identificar las estrategias que, según reportan los estudiantes, se han utilizado en la enseñanza de las teorías del desarrollo humano en cursos anteriores al curso Desarrollo Cognoscitivo del Niño de 0 a 7 años de la especialidad Educación Inicial del IPMJMSM.

La investigación constituye una investigación de campo, cuyos datos fueron recogidos directamente de la realidad. En este caso, se pretendió recolectar la información en el lugar donde la autora ejerce la docencia, para finalmente determinar el impacto de las estrategias basadas en la enseñanza estratégica como medio para favorecer el aprendizaje significativo de la Psicología del Desarrollo Humano, en los estudiantes de la especialidad Educación Inicial del IPMJMSM, en el período académico 2013 - I.

Así mismo, el carácter del presente estudio fue descriptivo-interpretativo por cuanto, apoyado en las ideas de Palella y Martins (2010), su propósito es el de interpretar realidades de hecho, incluyendo descripción, registro, análisis e interpretación de la naturaleza actual (p. 92).

Los sujetos que conformaron la muestra a investigar según Hernández-Sampier et al., (2014) es el conjunto de personas sobre las cuales se recolectan los datos. En este caso estuvieron representados por un grupo de 15 estudiantes, del curso mencionado y cuya administración estuvo a cargo de la autora del presente estudio y el muestreo a realizar fue no probabilístico intencional, por cuanto los participantes fueron escogidos con base en criterios preestablecidos por la investigadora (Arias, 2012).

Para desarrollar la presente investigación se hizo uso de las siguientes técnicas: La encuesta y la observación participante.

Palella y Martins (2010) definen a la encuesta como una técnica destinada a obtener datos de varias personas cuyas opiniones interesan al investigador a través de un listado de preguntas escritas (p. 123). Para los efectos del presente estudio, se aplicó la encuesta a la muestra seleccionada correspondiente al grupo 1 del curso DCN.

La observación es participante según el mismo autor, cuando el investigador se incluye en el grupo, hecho, fenómeno observado, para obtener información desde adentro (p. 118), en este caso la autora como docente del curso estuvo inmersa en el grupo investigado. 
Para recabar los datos de la presente investigación como instrumento el cuestionario, definido por Hernández-Sampier et al. (2014) como un conjunto de preguntas respecto a una o más variables a medir, fue utilizado en la presente investigación para llevar a cabo la encuesta. Este estuvo conformado por 36 ítems agrupado en 6 bloques de contenidos o pregunta global (ver Cuadro 1). Fue validado por expertos.

Cuadro 1. Cuestionario

Ítems

1.- Para abordar los contenidos sobre postulados teóricos y demás contenidos de Psicología, la mayoría de los docentes se han valido de ciertos métodos entre los cuales destacan:

\section{2.- Durante las sesiones la} mayoría de los docentes utilizan diversas técnicas entre las cuales predominan: cursos relacionados con la Psicología del desarrollo puedes identificar los siguientes momentos: a. Presentar el tema para luego de manera progresiva ir desglosándolo en partes para que se logrela comprensión del mismo en la sesión de clase.

b. Asignar al estudiantado la investigación varios puntos o temas para luego discutir en clase y establecer allí las conclusiones al respecto

c. Planteamiento de una situación problema para que los estudiantes a través de su resolución establezca relación entre los pasos seguidos, los resultados obtenidos y la comprensión del tema

d. Combinación de dos o más métodos entre los mencionados

a. Hace uso de clases magistrales.

b. Se vale de la demostración para dar a comprender el tema.

c. Promueve la discusión dirigida.

d. Propone talleres para desarrollar el contenido.

e. Plantea debates.

f. Asigna exposiciones que quedan a cargo de los estudiantes.

g. Propone puestas en común.

h. Propone galerías de concomimiento.

a. En las sesiones evidencias el momento de inicio centrando la atención del grupo.

b. Desarrollo de la sesión dividido en diferentes actividades que conllevan al logro del propósito de la clase.

c. Cierre de la sesión a través de actividades que sinteticen todo lo abordado (cierre cognitivo).

d. Cierre de la sesión a través de la concienciación sobre la utilidad y transferencia de lo aprendido a otros ámbitos (cierre metacognitivo).

e. Cierre de la sesión a través de actividades reflexivas que inviten a cada estudiante a un próximo encuentro o a continuar la revisión del contenido de forma independiente (Cierre afectivo) 


\section{Ítems}

4.- Dentro de las a. Orientados a una aplicación centrada en el estudiante, sus intereses, actividades llevadas a potencialidades y necesidades.

cabo por los docentes, b. Orientados a una aplicación centrada en el Docente, en lo que éste domina, conoce mayormente se $\quad$ y prefiere realizar o espera lograr. proponen eventos

c. Orientados a una aplicación centrada en el tema, contenido y la importancia del mismo dentro del pensum de estudios.

d. Orientados a una aplicación centrada en las actividades evaluativas, esperándose que se logren los objetivos sólo con la realización de la actividad evaluativa por parte de los estudiantes

5.- Durante el desarrollo

a. El docente evalúa solo el producto -resultado- posterior a la sesión.

de las sesiones con

b. El docente evalúa un producto asignado sin haber desarrollado el contenido correspondiente en una sesión con los estudiantes.

respecto a la evaluación puede señalar que:

c. El docente ofrece acompañamiento y monitoreo continuo de los procesos en todos los momentos de la sesión, haciendo preguntas y observando durante los mismos.

d. El docente propicia la autoevaluación.

e. El docente invita a la coevaluación.

f. Hay predominio de evaluaciones formativas.

g. Hay predominio de evaluaciones sumativas.

6.- Con respecto a los a. La pizarra y los marcadores.

medios y recursos b. El Rotafolios y láminas.

empleados por los c. Video beam y presentación ppt.

docentes en el desarrollo d. Franelógrafo.

de las sesiones de e. Retroproyector y filminas.

trabajo Ud. puede f. Periódicos murales o similares

señalar: $\quad$ g. Diagramas, mapas, esquemas, etc.

Para responder al problema planteado, la investigación se llevó a cabo en tres fases.

Fase I. Revisión teórica. Luego de realizar una revisión de la teoría relacionada con las estrategias de enseñanza y estrategias de aprendizaje, se procedió a elaborar los instrumentos que permitiera realizar un diagnóstico y establecer los elementos necesarios para el diseño de estrategias que se aplicarán.

Fase II. Diseño y aplicación de instrumento. Dicho diagnóstico se aplicó a los estudiantes al inicio del período académico en la segunda sesión de clases. Tuvo el propósito de indagar a través de la aplicación del cuestionario, acerca de las estrategias de enseñanza que los estudiantes refieran que han sido empleadas por los docentes para abordar los postulados de la psicología del desarrollo humano en los cursos anteriores.

Fase III. Análisis de resultados del diagnóstico. Luego de haber aplicado el instrumento a los estudiantes seleccionados, se procedió a analizar los resultados obtenidos para establecer el diagnóstico correspondiente y que permitió tener un punto de partida para llevar a cabo el diseño de estrategias en la etapa Il de la investigación.

Se procedió a registrar los resultados de la aplicación de cada una de las estrategias diseñadas para cada unidad del Curso DCN0613. Las impresiones obtenidas de la aplicación de cada una de las estrategias fueron registradas en un diario de campo llevado a cabo a través 
de la libreta de notas y adicionalmente también se llevaron a cabo registros a través del uso de cámara de fotografías. Este dispositivo incluido en el equipo telefónico de la autora.

\section{RESULTADOS}

Fase I. Revisión teórica. En esta fase de la investigación, se llevó a cabo una revisión detallada de los aspectos teóricos relacionados con las estrategias de enseñanza y la enseñanza estratégica para lograr definir los criterios y elementos necesarios para el diseño y construcción del instrumento de recolección de datos, en correspondencia plena con la matriz de operacionalización de variables.

Fase II. Diseño y aplicación del instrumento. Durante esta fase se inició y culminó el diseño de un cuestionario que constó de 36 ítems, distribuidos en seis bloques o grupos de preguntas globales cuyos rangos de respuestas fueron dispuestos para ser respondidos en función a la escala de frecuencia siguiente: Siempre-Frecuentemente- Muy pocas veces y Nunca.

El cuestionario, luego de ser diseñado, fue reproducido para ser aplicado a un grupo piloto conformado por 10 estudiantes de la especialidad de Educación Inicial, que contara con las mismas características de la muestra seleccionada. Esta aplicación o prueba piloto permitió, observar y detectar algunas ambigüedades en las instrucciones y realizar ajustes en función a la cantidad de ítems, eliminando cuatro preguntas que resultaban similares, quedando un total de seis preguntas.

Fase III. Análisis de resultados del diagnóstico. Posterior a la aplicación del instrumento a los estudiantes seleccionados, se procedió llevar a cabo el análisis respectivo a los resultados obtenidos los cuales arrojaron los datos necesarios y útiles para establecer el diagnóstico correspondiente. Tal diagnóstico permitió tener un punto de partida para llevar a cabo el diseño de estrategias en la etapa II de la investigación.
Pregunta Nro. 1. Para abordar los contenidos sobre postulados teóricos y demás contenidos de Psicología, la mayoría de los docentes se han valido de ciertos métodos entre los cuales destacan...

Se deja en evidencia que la mayoría de los estudiantes encuestados reportan que muy pocas veces o solo algunas veces hay actividades que responden a un método deductivo. Mientras que una mayoría considerable del $47 \%$ considera que los docentes frecuentemente recurren a acciones que responden a una metodología inductiva.

Por otra parte, reportan que muy pocas veces estos recurren a la propuesta de problemas y muy pocas veces observan la combinación de dos o más métodos para abordar sus contenidos durante el desarrollo de un curso.

Pregunta Nro. 2. Durante las sesiones la mayoría de los docentes utilizan diversas técnicas entre las cuales predominan...

Llama la atención que la mayoría de las encuestadas consideran que la técnica de la clase magistral, es utilizada por los docentes en sus prácticas educativas.

Adicionalmente, la mayoría de las encuestadas expresan que muy pocas veces es utilizada la demostración como técnica para abordar los contenidos en las sesiones de clase.

Por otra parte, la totalidad de las encuestadas, consideran que la discusión dirigida es utilizada por los docentes en su mayoría, contrario al debate que, de acuerdo con una mayoría bastante representativa no es una técnica que los docentes suelan incorporar en su práctica educativa al abordar los contenidos relacionados con la Psicología. Lo que permite determinar que el debate como técnica de enseñanza no es aprovechada por los docentes para facilitar y promover el logro de los objetivos. 
Resulta oportuno destacar que la mayoría de los encuestados considera que la técnica de la plenaria o puesta en común son usadas por los docentes pero muy pocas veces, dejando claro de esta manera que, aun siendo esta técnica provechosa para el momento de cierre de las estrategias de enseñanza que se apliquen, también es desaprovechada la técnica.

Haciendo un balance de los resultados de cada ítem, se puede afirmar que de acuerdo con lo que reportan las estudiantes encuestadas, de las técnicas propuestas las más utilizadas por los docentes son la clase magistral y la discusión dirigida y la menos utilizada resultó ser el debate.

\section{Pregunta Nro. 3. En las sesiones de los cursos relacionados con la Psicología del desarrollo puedes identificar los siguientes momentos...}

Se deja claro, en muchos casos, la ausencia de una estructura de secuencia didáctica en las sesiones de clase de acuerdo con lo reportado por los encuestados.

Primeramente, el momento de inicio, según las estudiantes encuestadas en un 53\% se evidencia en pocos casos, en contraste con un $34 \%$ que considera que se evidencia con frecuencia.

Con respecto al momento de desarrollo de las sesiones, conformado por diversas actividades que conlleven al logro del propósito, llama la atención que siendo este momento uno de los que generalmente siempre se evidencia, existe un porcentaje del $40 \%$ de las encuestadas que lo evidencian frecuentemente y no siempre como se espera en este caso.

En cuanto al momento de cierre, aunque la mayoría de las encuestadas, en este caso un $80 \%$, considera que muy pocas veces han evidenciado en las sesiones de clase un cierre cognitivo y un $13 \%$ manifiesta que lo ha evidenciado sólo algunas veces.

Aunado a lo anterior, pero en relación con el cierre metacognitivo, un $80 \%$ de las encuestadas consideró que nunca lo han evidenciado, lo cual constituye un hallazgo bastante significativo para la presente investigación, ya que adicionalmente un 13\% considera que muy pocas veces ha evidenciado este tipo de cierre, mientras un 7\% consideró que lo ha evidenciado pocas veces.

Del mismo modo resulta importante destacar que un 53\% de los estudiantes, afirma que nunca ha evidenciado un cierre afectivo, es decir, un cierre basado en actividades reflexivas, orientado a la introspección, orientado a que el estudiante establezca un vínculo afectivo y armónico con su grupo, con el docente y con lo aprendido, que invite a cada estudiante a un próximo encuentro posterior a las sesiones de clase, esto aunado a un $40 \%$ que manifiesta que ha podido evidenciarlo pero muy pocas veces y seguido de un $7 \%$ que manifiesta que sólo algunas veces ha podido evidenciarlo.

Estos hallazgos con respecto al momento de cierre resultan importantes, en virtud de que es en ese momento de cierre, sobre todo porque es en el cierre metacognitivo que se promueve en el estudiante la reflexión, autoevaluación de su propio proceso de aprendizaje, se promueve la concienciación de potencialidades, limitaciones y necesidades con respecto a sus propias estrategias de aprendizaje.

Pregunta Nro. 4. Dentro de las actividades llevadas a cabo por los docentes, mayormente se proponen eventos...

Se pudo determinar que todas las encuestadas consideran que las actividades no se centran en el estudiante, en sus necesidades, intereses y potencialidades, este resultado se distribuyó entre un 53\% que opinan que muy pocas veces las actividades están centradas en el estudiante, un $27 \%$ que opina que nunca se centran en el estudiante y un $20 \%$ considera que sólo algunas veces las actividades propuestas se centran en elestudiante.

Es importante destacar que las estudiantes reportan que las actividades propuestas por los 
docentes para abordar los contenidos están centradas mayormente en las actividades evaluativas y que adicionalmente se centran en el contenido y la importancia que este pueda tener dentro del pensum de estudio.

Pregunta Nro. 5. Durante el desarrollo de las sesiones con respecto a la evaluación, puede señalar que...

Casi la totalidad de las encuestadas considera que en lo que respecta a la evaluación, la mayoría de los docentes evalúan sólo los resultados, el $87 \%$ de las encuestadas consideran que los docentes evalúan el producto asignado sin haber trabajado el contenido de alguna manera con los estudiantes. Del mismo modo, gran parte de las encuestadas de la muestra considera que muy pocas veces dentro del desarrollo de las sesiones con respecto a la evaluación, el docente ofrece acompañamiento y monitoreo continuo de los procesos en todos los momentos de la sesión.

Aunque una buena parte consideraba que los docentes promueven la autoevaluación, dentro del proceso evaluativo, estos mismos consideran que se daba en muy pocas ocasiones igual que la coevaluación.

En relación con las formas de evaluación que predominan en los procesos de enseñanza, es importante destacar que dentro de los hallazgos de la investigación está el hecho de que una mínima parte de la muestra encuestada expresó que algunas veces ha observado una predominancia de la evaluación formativa dentro de las sesiones de clase, sin embargo una parte de éstos consideró que lo ha evidenciado muy pocas veces ha sido dejando claro que la mayor parte de los encuestados reporta que existe una predominancia en la evaluación sumativa y que la evaluación formativa no suele apreciarse lo suficiente dentro de las prácticas de los docentes.
Pregunta Nro. 6. Con respecto a los medios y recursos empleados por los docentes en el desarrollo de las sesiones de trabajo Ud. puede señalar...

Entre los medios y recursos se señalaron la pizarra y marcadores, el rotafolio y las láminas, el video beam y la presentación en PowerPoint, el franelógrafo, el retroproyector y las filminas, los periódicos murales o similares y los diagramas, mapas, esquemas, entre otros. Posterior a la revisión detallada de los resultados de cada ítem, se pudo determinar que de los mencionados recursos, entre los que más reportan las estudiantes encuestadas que utilizan los docentes se encuentran la presentación PowerPoint, la pizarra y los marcadores. El retroproyector es reportado como un recurso utilizado aunque muy pocas veces y del mismo modo el rotafolio y las láminas. No obstante, el resto de los recursos mencionados como el franelógrafo y el periódico mural o similares a éste, según reportan el 100\% de las encuestadas no son utilizados.

Después de haber llevado a cabo el diagnóstico producto de la encuesta aplicada a las estudiantes seleccionadas, se llegó a la segunda etapa de la investigación correspondiente al diseño de las estrategias de enseñanza.

Para consolidar esta etapa de diseño, se cotejaron los resultados obtenidos en función de establecer cómo se llevaban a cabo los procesos de enseñanza en los cursos anteriores al curso desarrollo Cognoscitivo del Niño de 0 a 7 años. Se logró determinar, a través del diagnóstico, que de acuerdo con lo que reportan los estudiantes no existe una claridad o conciencia por parte del docente acerca de cuáles son los métodos y técnicas más idóneos para abordar los contenidos que forman parte de suscursos. 
En este punto muchos de las estudiantes encuestadas refirieron que han observado que sus docentes se limitaban a asignar tareas que, generalmente, eran exposiciones que debían ser preparadas por los estudiantes sin realizar ningún tipo de explicación, por parte de los docentes, de los temas asignados.

Del mismo modo no se observa, según indican las encuestadas, una estructura en las sesiones de clase ajustada a los momentos y eventos propios de una estrategia de enseñanza, pues por lo general se evidencia el momento de desarrollo de la sesión, pero igualmente se reportan debilidades en el momento de inicio e incluso ausencia del momento de cierre, sobre todo en lo que se refiere a un cierre metacognitivo yafectivo.

Dentro de los aspectos diagnosticados a ser considerados para el diseño de estrategias, se encuentra el hecho de que el estudiante no percibe que las actividades sean planeadas tomando en consideración los intereses, necesidades y potencialidades del estudiante, sino que obedecen y se centran en las actividades evaluativas asignadas, y en ocasiones al tema y su importancia dentro del programa del curso y pensum de estudios.

Así mismo se pudo determinar que los estudiantes encuestados reportan que en el aspecto evaluativo, que constituye un eje importante en toda estrategia de enseñanza, se evidenció que existe una predominancia de la evaluación sumativa, desestimándose la evaluación formativa como factor vital del proceso de enseñanza, obviándose el monitoreo y acompañamiento continuo del docente que es tan necesario para garantizar que se alcancen los objetivos y se logren los propósitos planteados. Incluso según este diagnóstico se descuida la evaluación de procesos y se limita a la simple evaluación y calificación de resultados.

No hay una variedad o diversificación en cuanto a la selección de medios y recursos a utilizar para el desarrollo de las sesiones de clase, limitándose el docente al uso escaso de recursos tradicionales como la pizarra y el video beam con presentación PowerPoint, y dicho por las estudiantes muy escasamente y con presentaciones poco llamativas o interesantes, lo que hace pensar que este tipo de enseñanza resulta en todos los sentidos a considerar poco o nada estratégicas.

\section{CONCLUSIONES}

Para Cueva (2000) los métodos y las técnicas son seleccionados para orientar esas actividades conscientes y su respectiva estructura en correspondencia con el modelo de enseñanza y según sus propósitos. De allí que los docentes deban procurar, no solo variar las técnicas de sus clases, sino que deben obedecer a sus propósitos para promover verdaderas experiencias de aprendizaje que trasciendan el hecho educativo. Por lo que, si se toma en cuenta que en la investigación se evidenció una ausencia de conciencia metódica en la planeación de las sesiones de clase, se puede entender cómo esto influyó en la situación problema que dio origen al presente estudio. $Y$ como lo señala Martínez Valcárcel (2004), "no hay métodos ni buenos ni malos" (p. 2).

Otro punto importante fue la ausencia de una estructura de secuencia didáctica en las sesiones de clase, lo que no permitió que se llevaran a cabo los momentos propios de toda estrategia: Inicio; desarrollo y cierre. En el momento de inicio es donde se dan los procesos que se irán desarrollando en el transcurso de cualquier situación de enseñanza y de aprendizaje. Allí se presentan los objetivos y propósitos de la estrategia, se aprovecha el uso de organizadores previos, se genera una expectativa y se despierta el interés del estudiante. Durante el momento de cierre se contempla la realización de un cierre cognitivo, un cierre metacognitivo y un cierre afectivo. El primero representa la fase culminante de los procesos de enseñanza y de aprendizaje que se iniciaron en la sesión. El segundo favorece la reflexión acerca de los procesos, vivencias, habilidades, debilidades presentadas durante la sesión. El tercero está orientado a que el estudiante establezca un vínculo afectivo y 
armónico con su grupo, con el docente y con lo aprendido.

También se identificaron los aspectos en los que estaban centradas las actividades llevadas a cabo por los docentes para abordar los contenidos. En cuanto a esto, se pudo determinar que las actividades, generalmente, no se centran en el estudiante, en sus necesidades, intereses y potencialidades. Contrario a esto las actividades están centradas mayormente en las actividades evaluativas y que adicionalmente, en algunas pocas ocasiones se centran en el contenido y la importancia que este pueda tener dentro del pensum de estudio. Al respecto, Ausubel et al., (1987) conceden gran importancia a los contenidos con sentido para el que aprende, por lo que el docente debe tomar como base para el diseño de sus estrategias los intereses de sus estudiantes, sus potencialidades y sus necesidades, en función de que este le encuentre sentido a lo que está por aprender o está aprendiendo.

\section{REFERENCIAS}

Acevedo, A. (2002). Estrategias instruccionales para promover el dominio progresivo de los aspectos formales de la lengua escrita en alumnos de tercer grado de educación básica del Colegio "San Vicente De Paúl" de Guatire. XI Jornada de Investigación y II Encuentro de Postgrado del Instituto Pedagógico de Miranda "José Manuel Siso Martínez", UPEL

Arias, F. (2012). El Proyecto de Investigación. Introducción a la Metodología Científica. 5ta Edición. Caracas: Espíteme

Ausubel, D. (1976). Psicología Educativa. México: Trillas

Ausubel, D., Novak, J. y Hanesian, H. (1987). Psicología Educativa. México: Trillas

Cueva, V. (2000). Procedimientos de Estrategias, Técnicas y Métodos para Activar los Procesos de Aprendizaje. Una Interpretación Constructiva. Trujillo. [Material Mimeografiado]

Esteban, M. y Zapata, M. (2008). Estrategias de aprendizaje y elearning: Un apunte para la fundamentación del diseño educativo en los entornos virtuales de aprendizaje. Revista de Educación a Distancia. Recuperado de www.um.ead/red/19/
Flores, C. (2002). Estrategias instruccionales para la comprensión de lectura en estudiantes de educación integral en el Instituto Pedagógico de Miranda José Manuel Siso Martínez (Trabajo de ascenso). Universidad Pedagógica Experimental Libertador, Instituto Pedagógico de Miranda José Manuel Siso Martínez, Caracas, Venezuela

González, R. D. (2014). La enseñanza estratégica como medio para favorecer el aprendizaje de las teorías del desarrollo humano en estudiantes de la especialidad Educación Inicial (trabajo de maestría). Universidad Pedagógica Experimental Libertador, Miranda, Venezuela

Hernández, G. (1998). Paradigmas en Psicología de la Educación. México: Paidós

Hernández-Sampier, R., Fernández C., C. y Baptista L., P. (2014). Metodología de la Investigación. (6ta. ed.). México: McGrawHill Interamericana

Martínez Valcárcel, N. (2004). Los modelos de enseñanza y su aplicación en el aula. Universidad de Murcia. Recuperado de http://www.um.es/docencia/nicolas/menu/p ublicaciones/propias/docs/enciclo pediadidacticarev/modelos.pdf

Ministerio de Educación (1987). Manual del Docente. Tercera etapa de Educación Básica. Caracas: M.E

Palella, S. y Martins, F. (2010). Metodología de la investigación Cuantitativa. Caracas: Fondo Editorial de la Universidad Pedagógica Experimental Libertador FEDUPEL

Pérez Lo Presti, A. (2007). Psicología en Educación: Una visión contemporánea. Universidad de los Andes. Escuela de Educación. Mérida, Venezuela. En Revista EDUCERE. Recuperado de redalyc.uaemex.mx/pdf/356/35603906.pdf

Pozo, J. I., y Monereo, C. (1999). El aprendizaje estratégico. Madrid: Aula XXI, Santillana

Universidad Nacional Abierta (1983). Las técnicas de Enseñanza. Caracas: Autor

Universidad Pedagógica Experimental Libertador [UPEL] (1996a). Diseño Curricular de la Especialidad Educación Preescolar. Caracas: Autor

Universidad Pedagógica Experimental Libertador [UPEL] (1996b). Diseño Curricular componente de Formación Especializada de la Especialidad Educación Preescolar. Caracas: Autor 